\title{
A Class of Inexact Secant Algorithms with Line Search Filter Method for Nonlinear Programming
}

\author{
Zhujun Wang $\mathbb{1}^{1}$ and Li Cai ${ }^{2}$ \\ ${ }^{1}$ School of Computational Science and Electronics, Hunan Institute of Engineering, Xiangtan 411105, China \\ ${ }^{2}$ Shanghai Research Institute of Microwave Equipment, Shanghai 200063, China \\ Correspondence should be addressed to Zhujun Wang; 70127@hnie.edu.cn
}

Received 12 September 2021; Accepted 9 October 2021; Published 10 November 2021

Academic Editor: Guoqiang Wang

Copyright (c) 2021 Zhujun Wang and Li Cai. This is an open access article distributed under the Creative Commons Attribution License, which permits unrestricted use, distribution, and reproduction in any medium, provided the original work is properly cited.

\begin{abstract}
We propose a class of inexact secant methods in association with the line search filter technique for solving nonlinear equality constrained optimization. Compared with other filter methods that combine the line search method applied in most large-scale optimization problems, the inexact line search filter algorithm is more flexible and realizable. In this paper, we focus on the analysis of the local superlinear convergence rate of the algorithms, while their global convergence properties can be obtained by making an analogy with our previous work. These methods have been implemented in a Matlab code, and detailed numerical results indicate that the proposed algorithms are efficient for 43 problems from the CUTEr test set.
\end{abstract}

\section{Introduction}

This paper considers the following nonlinear equality constrained optimization problem:

$$
\begin{aligned}
& \min _{x \in \mathbb{R}^{n}} f(x), \\
& \text { s.t.c }(x)=0,
\end{aligned}
$$

where $f: \mathbb{R}^{n} \longrightarrow \mathbb{R}$ and $c: \mathbb{R}^{n} \longrightarrow \mathbb{R}^{m}$ are twice continuously differentiable.

After more than 20 years of development, filter algorithm has become an important method to solve constrained optimization problems and has been successfully applied to various fields of optimization (see [1-5]). In [6, 7], a line search filter Newton method is presented for (1). Local and global convergence properties of this method are analyzed. In particular, motivated by a multiobjective restructuring of constrained optimization problems, Eichfelder et al. [3] proposed a new filter approach, which utilizes the restructuring to identify the trade-off between constraint satisfaction and objective value. It is foreseeable that the filter method will find application in more diverse fields.

It is well known that the secant methods (two-step algorithms) are one of the most successful methods for solving problem (1) and have a main advantage which rests in the use of an orthogonal projection operator which is continuous. Fontecilla [8] showed that the six secant algorithms generate a sequence converging 2-step superlinearly under some suitable conditions.

When solving large-scale nonlinear programming problems or system of nonlinear equations, it would take too much time to get an exact solution in each iteration. An inexact Newton method was proposed in [9] to solve largescale system of nonlinear equations. Subsequently, an inexact SQP algorithm was presented in [2] to solve large-scale equality constrained optimization problems. Under suitable 
assumptions, the global convergence of inexact SQP steps was proved. These research results show that the use of inexact algorithm can save a lot of computing time.

Inspired by these ideas above, we propose a class of inexact secant methods in association with the line search filter technique, which has both global convergence and $q$ superlinear local convergence rate. These methods are globalized by line search and filter methods. In this work, we use Lagrangian function $\mathscr{L}$ as the objective function and $\|c(x)\|^{2}$ as the constraint violation. The filter method can suffer from the Maratos effect. The reason that Maratos effect appears is that the merit function used to judge whether the iteration point is good or bad is nonsmooth. If the merit function is smooth, Maratos effect can be avoided. Hence, we do not need to compute the second-order correction step which was used to overcome the Maratos effect.

The structure of this article is arranged as follows. Section 2 develops the algorithms. In Section 3, we mainly analyze the local superlinear convergence rate. The results of numerical experiences with the three algorithms are discussed in Section 4. Final conclusions are provided in Section 5.

\section{Algorithms}

In this paper, we denote the Euclidean vector or matrix norm by $\|\cdot\|, A(x):=\nabla c(x)$, and $g(x):=\nabla(x)$. The inexact secant algorithms are given by the following iterative scheme:

$$
\begin{gathered}
\lambda_{k+1}=U\left(x_{k}, \lambda_{k}, W_{k}\right), \\
W_{k} \widehat{u}_{k}=-\nabla_{x} \mathscr{L}\left(x_{k}, \lambda_{k+1}\right)+r_{k}, \\
u_{k}=P_{k} \widehat{u}_{k}, \\
v_{k}=-A_{k}^{\dagger} c_{k}, \\
d_{k}=u_{k}+v_{k}, \\
y_{k}=\nabla_{x} \mathscr{L}\left(x_{k}+u_{k}, \lambda_{k+1}\right)-\nabla_{x} \mathscr{L}\left(x_{k}, \lambda_{k+1}\right), \\
W_{k+1}=B\left(u_{k}, y_{k}, W_{k}\right), \\
x_{k+1}=x_{k}+d_{k},
\end{gathered}
$$

where $\left\|r_{k}\right\| \leq \eta_{k}\left\|c_{k}\right\|$ with $\eta_{k} \in[0, t]$ and $t \in(0,1), \mathscr{L}(x, \lambda)=$ $f(x)+\lambda^{T} c(x)$ for $\lambda \in \mathbb{R}^{m}$, and $B$ is the BFGS or the DFP secant update formula generating matrices $W_{k}$ approximating the Hessian of the Lagrangian at each $x_{k}$. There are many different multiplier updates; however, $\lambda_{k+1}$ in (2a) is chosen from the most commonly used multiplier updates: projection update, null-space update, and Newton update. $P(x)$ in (2c) can be either the orthogonal projection or the oblique projection depending on the choice of the multiplier update $\lambda_{k+1}$. $A_{k}^{\dagger}$ in (2d) is the pseudo-inverse of $A_{k}^{T}$. With different choices for $\lambda_{k+1}$ in (2a), $P(x)$ in (2c), and $A_{k}^{\dagger}$ in (2d), we get the following three algorithms: (i) ALG 1: $\lambda_{k+1}=-\left(A_{k}^{T} W_{k}^{-1} A_{k}\right)^{-1} A_{k}^{T} W_{k}^{-1} g_{k} \quad$ (nullspace update)

$$
\begin{aligned}
& P(x)=I \text { ( } I \text { is the identity matrix }) \\
& A_{k}^{\dagger}=W_{k}^{-1} A_{k}\left(A_{k}^{T} W_{k}^{-1} A_{k}\right)^{-1}
\end{aligned}
$$

(ii) ALG 2: $\lambda_{k+1}=-\left(A_{k}^{T} A_{k}\right)^{-1} A_{k}^{T} g_{k}$ (projection update) $P(x)=I-W^{-1} A(x)\left[A(x)^{T} W^{-1} A(x)\right]^{-1} A(x)^{T}$ (oblique projection)

$$
A_{k}^{\dagger}=A_{k}\left(A_{k}^{T} A_{k}\right)^{-1}
$$

(iii) ALG 3: $\quad \lambda_{k+1}=\left(A_{k}^{T} W_{k}^{-1} A_{k}\right)^{-1}\left(c_{k}-A_{k}^{T} W_{k}^{-1} g_{k}\right)$ (Newton update)

$$
P(x)=I-A(x)\left[A(x)^{T} A(x)\right]^{-1} A(x)^{T} \text { (orthog- }
$$

onal projection)

$$
A_{k}^{\dagger}=W_{k}^{-1} A_{k}\left(A_{k}^{T} W_{k}^{-1} A_{k}\right)^{-1}
$$

To get the next iterate, the line search filter method described in Section 4.1 of the literature [6] is used to determine a step size $\alpha_{k} \in(0,1]$. The Lagrangian function $\mathscr{L}(x, \lambda)$ measures optimality and the constraint violation $h(x)$ measures feasibility, where $h(x)=1 / 2\|c(x)\|^{2}$. The class of complete algorithms can be stated as follows.

\section{Convergence Analysis}

The global convergence analysis of the algorithms in Section 3 of [10] still holds. The only point that needs special attention is the proof of Lemma 3.2 in [10]. This lemma proves that the search direction is a sufficient descent direction for $\mathscr{L}$ at the points that are sufficiently close to feasible and nonoptimal. The analysis of Lemma 3.2 in [10] then holds The analysis in Lemma 2 then holds with replacing "Steps 1 and 4" in Algorithm 1 by "ALG 1," "Steps 2 and 5" in Algorithm 1 by "ALG 2," and "Steps 3 and 6" in Algorithm 1 by "ALG 3" where appropriate.

Next, we analyze the local convergence rate of the proposed algorithms. Assume that $\left\{x_{k}\right\}$ and $\left\{\lambda_{k}\right\}$ generated by the class of algorithms converge to local solution $x^{*}$ and $\lambda^{*}$, respectively. In addition, they are contained in a convex set $\Omega$ Furthermore, we give the following assumptions.

\subsection{Assumptions $L$}

L1. The functions $f$ and $c$ are twice continuously differentiable in a neighborhood $\Omega$ of $x^{*}$.

L2. $x^{*}$ and $\lambda^{*}$ satisfy $\nabla_{x} \mathscr{L}\left(x^{*}, \lambda^{*}\right)=0$.

L3. $A\left(x^{*}\right)$ is full column rank.

L4. There exists a constant $\kappa>0$ such that $u^{T} W_{k} u \geq \kappa\|u\|^{2}$ for $A_{k}^{T} u=0$ and $W^{*}$ is positive definite on the null space of $A\left(x^{*}\right)^{T}$. The sequence $\left\{W_{k}\right\}$ is uniformly bounded.

$L 5 . W_{k}$ satisfies that

$$
\lim _{k \rightarrow \infty} \frac{\left\|\left[W_{k}-\nabla_{x x}^{2} \mathscr{L}\left(x_{k}, \lambda_{k+1}\right)\right] d_{k}\right\|}{\left\|d_{k}\right\|}=0 .
$$

For the sake of local convergence analysis, we define a penalty function 


$$
\phi_{\omega}(x, \lambda):=f(x)+\lambda^{T} c(x)+\omega h(x) .
$$

The local linearized approximation of the penalty function is

$$
\begin{aligned}
q_{\omega}\left(x_{k}, d, \lambda_{k}, d^{\lambda}\right) & =f_{k}+g_{k}^{T} d+\left(\lambda_{k}+d^{\lambda}\right)^{T}\left(c_{k}+A_{k}^{T} d\right) \\
& +\frac{1}{2} u^{T} W_{k} u+\frac{\omega}{2}\left\|c_{k}+A_{k}^{T} d\right\|^{2} .
\end{aligned}
$$

Lemma 1. If Assumptions $L$ hold and $\eta_{k}=O\left(\left\|d_{k}\right\|\right)$, then there exists a constant $\widetilde{\gamma}_{\omega}>0$ such that when $\omega \geq \widetilde{\gamma}_{\omega}$,

$$
q_{\omega}\left(x_{k}, 0, \lambda_{k}, 0\right)-q_{\omega}\left(x_{k}, d_{k}, \lambda_{k}, d_{k}^{\lambda}\right) \geq 0 .
$$

Proof. From (2d) and the assumption of global convergence, we can obtain that $v_{k}=O\left(\left\|c_{k}\right\|\right)$. (5) implies that

$$
\begin{aligned}
q_{\omega}\left(x_{k}, 0, \lambda_{k}, 0\right)-q_{\omega}\left(x_{k}, d_{k}, \lambda_{k}, d_{k}^{\lambda}\right) & =\frac{\omega}{2}\left\|c_{k}\right\|^{2}+\lambda_{k}^{T} c_{k} \\
& -g_{k}^{T} d_{k}-\frac{1}{2} u_{k}^{T} W_{k} u_{k} .
\end{aligned}
$$

For ALG 1, we have that

$$
\begin{aligned}
u_{k}^{T} W_{k} u_{k} & =u_{k}^{T}\left[-g_{k}+A_{k}\left(A_{k}^{T} W_{k}^{-1} A_{k}\right)^{-1} A_{k}^{T} W_{k}^{-1} g_{k}+r_{k}\right] \\
& =-u_{k}^{T} g_{k}+r_{k}^{T} W_{k}^{-1} A_{k}\left(A_{k}^{T} W_{k}^{-1} A_{k}\right)^{-1} A_{k}^{T} W_{k}^{-1} g_{k}+u_{k}^{T} r_{k},
\end{aligned}
$$

and

$g_{k}^{T} u_{k}=-u_{k}^{T} W_{k} u_{k}+g_{k}^{T} W_{k}^{-1} A_{k}\left(A_{k}^{T} W_{k}^{-1} A_{k}\right)^{-1} A_{k}^{T} W_{k}^{-1} r_{k}+r_{k}^{T} u_{k}$.

Since $\eta_{k}=O\left(\left\|d_{k}\right\|\right)$, we have $\left\|r_{k}\right\|=O\left(\left\|u_{k}\right\|\left\|c_{k}\right\|\right)$ $+\mathrm{O}\left(\left\|c_{k}\right\|^{2}\right)$. Hence, from (2a)-(2h), (7), (9), and L4, we can get that

$$
\begin{aligned}
q_{\omega}\left(x_{k}, 0, \lambda_{k}, 0\right)-q_{\omega}\left(x_{k}, d_{k}, \lambda_{k}, d_{k}^{\lambda}\right) & =\frac{\omega}{2}\left\|c_{k}\right\|^{2}+\frac{1}{2} u_{k}^{T} W_{k} u_{k} \\
& +O\left(\left\|u_{k}\right\|\left\|c_{k}\right\|\right)+O\left(\left\|c_{k}\right\|^{2}\right) \\
& \geq\left(\frac{\omega}{2}-\kappa_{3}\right)\left\|c_{k}\right\|^{2}-\kappa_{4}\left\|u_{k}\right\|\left\|c_{k}\right\| \\
& +\frac{1}{2} \kappa\left\|u_{k}\right\|^{2},
\end{aligned}
$$

where $\kappa_{3}, \kappa_{4}>0$. It follows with $\theta_{k}:=\left\|u_{k}\right\| /\left\|c_{k}\right\|$ that

$$
\begin{aligned}
\frac{1}{\left\|c_{k}\right\|^{2}}\left[q_{\omega}\left(x_{k}, 0, \lambda_{k}, 0\right)-q_{\omega}\left(x_{k}, d_{k}, \lambda_{k}, d_{k}^{\lambda}\right)\right] & \geq\left(\frac{\omega}{2}-\kappa_{3}\right)-\kappa_{4} \theta_{k}+\frac{1}{2} \kappa \theta_{k}^{2} \\
& =\frac{1}{2} \kappa\left(\theta_{k}-\frac{\kappa_{4}}{\kappa}\right)^{2}+\frac{\omega}{2}-\kappa_{3}-\frac{\kappa_{4}^{2}}{2 \kappa} .
\end{aligned}
$$

Let $\tilde{\gamma}_{\omega}:=2 \kappa_{3}+\kappa_{4}^{2} / \kappa$. Then, (6) holds for $\omega \geq \tilde{\gamma}_{\omega}$.

For ALG 2, we obtain that

$$
\begin{aligned}
u_{k} & =P_{k} \widehat{u}_{k}=-W_{k}^{-1} g_{k}+W_{k}^{-1} A_{k}\left(A_{k}^{T} W_{k}^{-1} A_{k}\right)^{-1} A_{k}^{T} W_{k}^{-1} g_{k}+P_{k} W_{k}^{-1} r_{k}, \\
u_{k}^{T} W_{k} u_{k} & =u_{k}^{T}\left(-\nabla_{x} \mathscr{L}+r_{k}\right)-u_{k}^{T} A_{k}\left(A_{k}^{T} W_{k}^{-1} A_{k}\right)^{-1} A_{k}^{T} W_{k}^{-1}\left(-\nabla_{x} \mathscr{L}+r_{k}\right) \\
& -u_{k}^{T} g_{k}+u_{k}^{T} r_{k}+u_{k}^{T} A_{k}\left(A_{k}^{T} W_{k}^{-1} A_{k}\right)^{-1} A_{k}^{T} W_{k}^{-1} g_{k}-u_{k}^{T} A_{k}\left(A_{k}^{T} W_{k}^{-1} A_{k}\right)^{-1} A_{k}^{T} W_{k}^{-1} r_{k} .
\end{aligned}
$$


Hence,

$$
g_{k}^{T} u_{k}=-u_{k}^{T} W_{k} u_{k}+r_{k}^{T} u_{k}-r_{k}^{T} W_{k}^{-1} A_{k}\left(A_{k}^{T} W_{k}^{-1} A_{k}\right)^{-1} A_{k}^{T} u_{k} .
$$

Using (7), (13), and L4, we have that

$$
\begin{aligned}
q_{\omega}\left(x_{k}, 0, \lambda_{k}, 0\right) & -q_{\omega}\left(x_{k}, d_{k}, \lambda_{k}, d_{k}^{\lambda}\right) \\
& =\frac{\omega}{2}\left\|c_{k}\right\|^{2}+\frac{1}{2} u_{k}^{T} W_{k} u_{k}+\lambda_{k}^{T} c_{k}-r_{k}^{T} u_{k} \\
& +r_{k}^{T} W_{k}^{-1} A_{k}\left(A_{k}^{T} W_{k}^{-1} A_{k}\right)^{-1} A_{k}^{T} u_{k}-g_{k}^{T} v_{k} \\
& =\frac{\omega}{2}\left\|c_{k}\right\|^{2}+\frac{1}{2} u_{k}^{T} W_{k} u_{k}+O\left(\left\|u_{k}\right\|\left\|c_{k}\right\|\right)+O\left(\left\|c_{k}\right\|^{2}\right) \\
& \geq\left(\frac{\omega}{2}-\widehat{\kappa}_{3}\right)\left\|c_{k}\right\|^{2}-\widehat{\kappa}_{4}\left\|u_{k}\right\|\left\|c_{k}\right\|+\frac{1}{2} \kappa\left\|u_{k}\right\|^{2},
\end{aligned}
$$

where $\widehat{\kappa}_{3}, \widehat{\kappa}_{4}>0$. Define $\theta_{k}:=\left\|u_{k}\right\| /\left\|c_{k}\right\|$, and (14) then implies that

$$
\begin{aligned}
\frac{1}{\left\|c_{k}\right\|^{2}}\left[q_{\omega}\left(x_{k}, 0, \lambda_{k}, 0\right)-q_{\omega}\left(x_{k}, d_{k}, \lambda_{k}, d_{k}^{\lambda}\right)\right] & \geq \frac{1}{2} \kappa\left(\theta_{k}-\frac{\widehat{\kappa}_{4}}{\kappa}\right)^{2} \\
& +\frac{\omega}{2}-\widehat{\kappa}_{3}-\frac{\widehat{\kappa}_{4}^{2}}{2 \kappa} .
\end{aligned}
$$

Let $\tilde{\gamma}_{\omega}:=2 \widehat{\kappa}_{3}+\widehat{\kappa}_{4}^{2} / \kappa$. Then, (6) holds for $\omega \geq \widetilde{\gamma}_{\omega}$. For ALG 3, (2a)-(2h) imply that

$$
\begin{aligned}
u_{k} & =\left(I-A_{k}\left[A_{k}^{T} A_{k}\right]^{-1} A_{k}^{T}\right) \widehat{u}_{k} \\
& =-W_{k}^{-1} g_{k}+W_{k}^{-1} A_{k}\left(A_{k}^{T} W_{k}^{-1} A_{k}\right)^{-1} A_{k}^{T} W_{k}^{-1} g_{k}-W_{k}^{-1} A_{k}\left(A_{k}^{T} W_{k}^{-1} A_{k}\right)^{-1} c_{k}+A_{k}\left(A_{k}^{T} A_{k}\right)^{-1} c_{k}+P_{k} W_{k}^{-1} r_{k} \\
& =\widehat{u}_{k}-W_{k}^{-1} r_{k}-v_{k}+P_{k} W_{k}^{-1} r_{k}, \\
u_{k}^{T} W_{k} u_{k} & =u_{k}^{T} W_{k} \widehat{u}_{k}-u_{k}^{T} r_{k}-u_{k}^{T} W_{k} v_{k}+u_{k}^{T} W_{k} P_{k} W_{k}^{-1} r_{k}=-u_{k}^{T} g_{k}-u_{k}^{T} W_{k} v_{k}+u_{k}^{T} W_{k} P_{k} W_{k}^{-1} r_{k}
\end{aligned}
$$

and

$$
g_{k}^{T} u_{k}=-u_{k}^{T} W_{k} u_{k}-u_{k}^{T} W_{k} v_{k}+u_{k}^{T} W_{k} P_{k} W_{k}^{-1} r_{k} .
$$

From (7), (17), and L4, we deduce that

$$
\begin{aligned}
q_{\omega}\left(x_{k}, 0, \lambda_{k}, 0\right) & -q_{\omega}\left(x_{k}, d_{k}, \lambda_{k}, d_{k}^{\lambda}\right) \\
& =\frac{\omega}{2}\left\|c_{k}\right\|^{2}+\lambda_{k}^{T} c_{k}+\frac{1}{2} u_{k}^{T} W_{k} u_{k}+u_{k}^{T} W_{k} v_{k} \\
& -u_{k}^{T} W_{k} P_{k} W_{k}^{-1} r_{k}-g_{k}^{T} v_{k} \\
& =\frac{\omega}{2}\left\|c_{k}\right\|^{2}+\frac{1}{2} u_{k}^{T} W_{k} u_{k} \\
& +O\left(\left\|u_{k}\right\|\left\|c_{k}\right\|\right)+O\left(\left\|c_{k}\right\|^{2}\right) .
\end{aligned}
$$

Similarly, we can also prove that there exists $\widetilde{\gamma}_{\omega}$ such that (6) holds for $\omega \geq \widetilde{\gamma}_{\omega}$.
Lemma 2. Suppose Assumptions L hold and $\eta_{k}=O\left(\left\|d_{k}\right\|\right)$. There exists a neighborhood $\mathscr{B}_{1}$ of $x^{*}$ such that if the first equation in Step 6 in Algorithm 1 holds for $\alpha_{k, l}=1$, then the trial step is accepted by the Armijo condition (the second equation in Step 6 in Algorithm 1) for $x_{k} \in \mathscr{B}_{1}$.

Proof. If the first equation in Step 6 in Algorithm 1 holds, $s_{\mathscr{L}}>2 s_{h}$ implies that

$$
\begin{aligned}
\frac{1}{2}\left\|c_{k}\right\|^{2} & =h\left(x_{k}\right)<\tau_{1}^{-1 / s_{h}}\left(-m_{k}(1)\right)^{s_{\mathscr{L}} / s_{h}} \\
& <\tau_{1}^{-1 / s_{h}}\left(-g_{k}^{T} d_{k}+\lambda_{k}^{T} c_{k}\right)^{s_{\mathscr{L}} / s_{h}} \\
& =\tau_{1}^{-1 / s_{h}}\left(-g_{k}^{T} u_{k}+O\left(\left\|v_{k}\right\|^{2}\right)\right)^{s_{\mathscr{L}} / s_{h}} \\
& =o\left(\left\|u_{k}\right\|^{2}\right) .
\end{aligned}
$$

By (9), (13), (17), and $r_{k}, v_{k}=O\left(\left\|c_{k}\right\|\right)$, we can easily get 
(1) Initialize: choose starting point $x_{0}, \lambda_{0}$, and $W_{0}=I$. Set constants $\beta \in(0,1 / 2), \gamma_{h}, \gamma_{\mathscr{L}} \in(0,1), t \in\left(0,1-\gamma_{h} / 1+\gamma_{h}\right), \tau_{1}, \nu>0$, $s_{h}>1, s_{\mathscr{L}}>2 s_{h}, \gamma_{\alpha} \in(0,1], \varsigma \in(0,1)$, and $\varepsilon>0$. Choose an initial filter $\mathscr{F}_{0}:=\left\{(h, \mathscr{L}) \in \mathbb{R}^{2}: h \geq \nu\right\}$ and let $k:=0$.

(2) If $\left\|\left(\begin{array}{c}\nabla_{x} \mathscr{L}\left(x_{k}, \lambda_{k}\right) \\ c_{k}\end{array}\right)\right\|<\varepsilon$, then stop.

(3) Evaluate $f_{k}, g_{k}, c_{k}$, and $A_{k}$. Then, compute the multiplier $\lambda_{k+1}=U\left(x_{k}, \lambda_{k}, W_{k}\right)$ from (2a). Find an inexact step $d_{k}$ from (2b)-(2e). Compute $d_{k}^{\lambda}=\lambda_{k+1}-\lambda_{k}$. If this system $((2 \mathrm{a})-(2 \mathrm{~h}))$ is too ill-conditioned, go to Step 9 .

(4) Set $\alpha_{k, 0}=1$ and $l=0$.

(5) If $\alpha_{k, l}<\alpha_{k}^{\min }$ with

$$
\alpha_{k}^{\min }:=\gamma_{\alpha} \cdot \begin{cases}\min \left\{\gamma_{h},\left(\gamma_{\mathscr{L}} h_{k} /\left(-g_{k}^{T} d_{k}+\lambda_{k}^{T} c_{k}-\left|\left(d_{k}^{\lambda}\right)^{T} c_{k}\right|\right)\right),\left(\tau_{1} h_{k}^{s_{h}} /\left(\left(-g_{k}^{T} d_{k}+\lambda_{k}^{T} c_{k}-\left|\left(d_{k}^{\lambda}\right)^{T} c_{k}\right|\right)^{s_{\mathscr{L}}}\right)\right)\right\} & \text { if } g_{k}^{T} d_{k}-\lambda_{k}^{T} c_{k}+\left|\left(d_{k}^{\lambda}\right)^{T} c_{k}\right|<0 \\ \text { otherwise }\end{cases}
$$

go to Step 9. Otherwise, if $\left(h\left(x_{k}+\alpha_{k, l} d_{k}\right), \mathscr{L}\left(x_{k}+\alpha_{k, l} d_{k}, \lambda_{k}+\alpha_{k, l} d_{k}^{\lambda}\right)\right) \in \mathscr{F}_{k}$, then

go to Step 7.

(6) Define $m_{k}(\alpha)$ : $=\alpha g_{k}^{T} d_{k}-\alpha \lambda_{k}^{T} c_{k}+\alpha(1-\alpha) c_{k}^{T} d_{k}^{\lambda}$. If

$m_{k}\left(\alpha_{k, l}\right)<0,\left[-m_{k}\left(\alpha_{k, l}\right)\right]^{s_{\mathscr{L}}} \alpha_{k, l}^{1-s_{\mathscr{L}}}>\tau_{1} h\left(x_{k}\right)^{s_{h}}$ and

$\mathscr{L}\left(x_{k}+\alpha_{k, l} d_{k}, \lambda_{k}+\alpha_{k, l} d_{k}^{\lambda}\right) \leq \mathscr{L}\left(x_{k}, \lambda_{k}\right)+\beta m_{k}\left(\alpha_{k, l}\right)$

hold, set $x_{k+1}$ : $=x_{k}+\alpha_{k, l} d_{k}$ and go to Step 8. If the first equation in Step 6 in Algorithm 1 does not hold, then if $h\left(x_{k}+\alpha_{k, l} d_{k}\right) \leq\left(1-\gamma_{h}\right) h\left(x_{k}\right)$

or

$\mathscr{L}\left(x_{k}+\alpha_{k, l} d_{k}, \lambda_{k+1}\right) \leq \mathscr{L}\left(x_{k}, \lambda_{k}\right)-\gamma_{\mathscr{L}} h\left(x_{k}\right)$

holds, set $x_{k+1}:=x_{k}+\alpha_{k, l} d_{k}$ and go to Step 8 .

(7) Set $\alpha_{k, l+1}=\varsigma \alpha_{k, l}$ and $l \leftarrow l+1$. Go to Step 5 .

(8) Set $\alpha_{k}:=\alpha_{k, l}$. If the first equation in Step 6 in Algorithm 1 does not hold for $\alpha_{k}$, augment the filter by $\mathscr{F}_{k+1}=\mathscr{F}_{k} \cup\left\{(h, \mathscr{L}) \in \mathbb{R}^{2}: h \geq\left(1-\gamma_{h}\right) h\left(x_{k}\right)\right.$ and $\left.\mathscr{L} \geq \mathscr{L}\left(x_{k}, \lambda_{k}\right)-\gamma_{\mathscr{L}} h\left(x_{k}\right)\right\}$

otherwise, set $\mathscr{F}_{k+1}$ : $=\mathscr{F}_{k}$. Go to Step 10 .

(9) Find a new point $x_{k+1}$ that satisfies the following conditions:

(a) $h\left(x_{k}\right)$ is greater than $h\left(x_{k+1}\right)$

(b) The third and fourth equations in Step 6 in Algorithm 1 hold for $x_{k+1}$

(c) $\left(h\left(x_{k+1}\right), \mathscr{L}\left(x_{k+1}, \lambda_{k+1}\right)\right) \notin \mathscr{F}_{k}$

Augment the filter by the equation in Step 8 in Algorithm 1. Go to Step 10.

(10) Compute $y_{k}=P_{k}\left[\nabla_{x} \mathscr{L}\left(x_{k}+u_{k}, \lambda_{k}\right)-\nabla_{x} \mathscr{L}\left(x_{k}, \lambda_{k}\right)\right]$ and update $W_{k}$ by $(2 \mathrm{~g})$. Set $k:=k+1$ and go to Step 2 .

Algorithm 1: ISLSF.

$$
\begin{aligned}
g_{k}^{T} d_{k} & =-u_{k}^{T} W_{k} u_{k}+O\left(\left\|u_{k}\right\|\left\|c_{k}\right\|\right)+O\left(\left\|r_{k}\right\|\right) \\
& =-u_{k}^{T} W_{k} u_{k}+o\left(\left\|u_{k}\right\|^{2}\right) .
\end{aligned}
$$

Hence, by Assumption L5, (19), and (20), we see that

$$
\begin{aligned}
\mathscr{L}\left(x_{k}, \lambda_{k}\right) & -\mathscr{L}\left(x_{k}+d_{k}, \lambda_{k}+d_{k}^{\lambda}\right)+\beta\left(g_{k}^{T} d_{k}-\lambda_{k}^{T} c_{k}\right) \\
& =\lambda_{k}^{T} c_{k}-g_{k}^{T} d_{k}-\frac{1}{2} d_{k}^{T} \nabla^{2} f\left(x_{k}\right) d_{k}-\left(\lambda_{k}+d_{k}^{\lambda}\right)^{T} c\left(x_{k}+d_{k}\right)+\beta\left(g_{k}^{T} d_{k}-\lambda_{k}^{T} c_{k}\right)+o\left(\left\|d_{k}\right\|^{2}\right) \\
& =(\beta-1) g_{k}^{T} d_{k}+(1-\beta) \lambda_{k}^{T} c_{k}-\frac{1}{2} d_{k}^{T} \nabla^{2} f\left(x_{k}\right) d_{k}-\frac{1}{2} d_{k}^{T}\left[\sum_{i=1}^{m} \lambda_{k, i} \nabla^{2} c_{i}\left(x_{k}\right)\right] d_{k}+o\left(\left\|d_{k}\right\|^{2}\right) \\
& =(1-\beta) u_{k}^{T} W_{k} u_{k}-\frac{1}{2}\left(u_{k}^{T} W_{k} u_{k}+2 u_{k}^{T} W_{k} v_{k}+v_{k}^{T} W_{k} v_{k}\right)+o\left(\left\|u_{k}\right\|\right)+o\left(\left\|d_{k}\right\|^{2}\right) \\
& =\left(\frac{1}{2}-\beta\right) u_{k}^{T} W_{k} u_{k}+O\left(\left\|c_{k}\right\|\right)+o\left(\left\|u_{k}\right\|^{2}\right)=\left(\frac{1}{2}-\beta\right) u_{k}^{T} W_{k} u_{k}+o\left(\left\|u_{k}\right\|^{2}\right) .
\end{aligned}
$$


From Assumption L4, $\beta \in(0,1 / 2)$, and we can conclude that the Armijo condition (the second equation in Step 6 in Algorithm 1) holds for $k$ sufficiently large.
Lemma 3. Suppose Assumptions $L$ hold. Choose $\eta_{k}=O\left(\left\|d_{k}\right\|\right)$. There exist a neighborhood $\mathscr{B}_{2} \subseteq \mathscr{B}_{1}$ and a constant $\gamma_{\omega}>0$ such that for $\omega \geq \gamma_{\omega}$ and $x_{k} \in \mathscr{B}_{2}$,

Next, we present a result which is necessary for the local convergence.

$$
\phi_{\omega}\left(x_{k}, \lambda_{k}\right)-\phi_{\omega}\left(x_{k}+d_{k}, \lambda_{k}+d_{k}^{\lambda}\right) \geq \frac{1+\gamma_{h}}{2}\left[q_{\omega}\left(x_{k}, 0, \lambda_{k}, 0\right)-q_{\omega}\left(x_{k}, d_{k}, \lambda_{k}, d_{k}^{\lambda}\right)\right]
$$

Proof. From (20) and $v_{k}=O\left(\left\|c_{k}\right\|\right)$, we have that

$$
\begin{aligned}
\phi_{\omega}\left(x_{k}, \lambda_{k}\right) & -\phi_{\omega}\left(x_{k}+d_{k}, \lambda_{k}+d_{k}^{\lambda}\right) \\
& =f_{k}+\lambda_{k}^{T} c_{k}+\frac{\omega}{2}\left\|c_{k}\right\|^{2}-f\left(x_{k}+d_{k}\right)-\left(\lambda_{k}+d_{k}^{\lambda}\right)^{T} c\left(x_{k}+d_{k}\right)-\frac{\omega}{2}\left\|c\left(x_{k}+d_{k}\right)\right\|^{2} \\
& =\lambda_{k}^{T} c_{k}+\frac{\omega}{2}\left\|c_{k}\right\|^{2}-g_{k}^{T} d_{k}-\frac{1}{2} d_{k}^{T} \nabla^{2} f\left(x_{k}\right) d_{k}-\lambda_{k}^{T} c\left(x_{k}+d_{k}\right)+o\left(\left\|d_{k}\right\|^{2}\right) \\
& =\frac{\omega}{2}\left\|c_{k}\right\|^{2}-g_{k}^{T} d_{k}+\lambda_{k}^{T} c_{k}-\frac{1}{2} d_{k}^{T} \nabla^{2} f\left(x_{k}\right) d_{k}-\frac{1}{2} d_{k}^{T}\left[\sum_{i=1}^{m} \lambda_{k, i} \nabla^{2} c_{i}\left(x_{k}\right)\right] d_{k}+o\left(\left\|d_{k}\right\|^{2}\right) \\
& =\frac{\omega}{2}\left\|c_{k}\right\|^{2}+u_{k}^{T} W_{k} u_{k}-\frac{1}{2} d_{k}^{T} W_{k} d_{k}+O\left(\left\|u_{k}\right\|\left\|c_{k}\right\|\right)+O\left(\left\|c_{k}\right\|^{2}\right)+o\left(\left\|d_{k}\right\|^{2}\right) \\
& =\frac{\omega}{2}\left\|c_{k}\right\|^{2}+u_{k}^{T} W_{k} u_{k}-\frac{1}{2}\left(u_{k}^{T} W_{k} u_{k}+2 u_{k}^{T} W_{k} v_{k}+v_{k}^{T} W_{k} v_{k}\right)+O\left(\left\|u_{k}\right\|\left\|c_{k}\right\|\right)+O\left(\left\|c_{k}\right\|^{2}\right)+o\left(\left\|d_{k}\right\|^{2}\right) \\
& =\frac{\omega}{2}\left\|c_{k}\right\|^{2}+\frac{1}{2} u_{k}^{T} W_{k} u_{k}+O\left(\left\|u_{k}\right\|\left\|c_{k}\right\|\right)+O\left(\left\|c_{k}\right\|^{2}\right)+o\left(\left\|u_{k}\right\|^{2}\right) .
\end{aligned}
$$

Hence, from Assumption L2, (10), (14), (18), (23), and $\gamma_{h} \in(0,1)$, we obtain that

$$
\begin{aligned}
\phi_{\omega}\left(x_{k}, \lambda_{k}\right) & -\phi_{\omega}\left(x_{k}+d_{k}, \lambda_{k}+d_{k}^{\lambda}\right)-\frac{1+\gamma_{h}}{2}\left[q_{\omega}\left(x_{k}, 0, \lambda_{k}, 0\right)-q_{\omega}\left(x_{k}, d_{k}, \lambda_{k}, d_{k}^{\lambda}\right)\right] \\
& =\frac{\omega}{2}\left\|c_{k}\right\|^{2}+\frac{1}{2} u_{k}^{T} W_{k} u_{k}+O\left(\left\|u_{k}\right\|\left\|c_{k}\right\|\right)+O\left(\left\|c_{k}\right\|^{2}\right)+o\left(\left\|u_{k}\right\|^{2}\right)-\frac{1+\gamma_{h}}{2}\left[\frac{\omega}{2}\left\|c_{k}\right\|^{2}+\frac{1}{2} u_{k}^{T} W_{k} u_{k}+O\left(\left\|u_{k}\right\|\left\|c_{k}\right\|\right)+O\left(\left\|c_{k}\right\|^{2}\right)\right] \\
& =\frac{1-\gamma_{h}}{4} \omega\left\|c_{k}\right\|^{2}+\frac{1-\gamma_{h}}{4} u_{k}^{T} W_{k} u_{k}+O\left(\left\|u_{k}\right\|\left\|c_{k}\right\|\right)+O\left(\left\|c_{k}\right\|^{2}\right)+o\left(\left\|u_{k}\right\|^{2}\right) \\
& \geq \frac{1-\gamma_{h}}{4} \kappa\left\|u_{k}\right\|^{2}-\kappa_{5}\left\|u_{k}\right\|\left\|c_{k}\right\|+\left(\frac{1-\gamma_{h}}{4} \omega-\kappa_{6}\right)\left\|c_{k}\right\|^{2}
\end{aligned}
$$

where $\kappa_{5}, \kappa_{6}>0$. Set $\theta_{k}:=\left\|u_{k}\right\| /\left\|c_{k}\right\|$. Therefore, we can get 


$$
\begin{aligned}
& \frac{1}{\left\|c_{k}\right\|^{2}}\left\{\phi_{\omega}\left(x_{k}, \lambda_{k}\right)-\phi_{\omega}\left(x_{k}+d_{k}, \lambda_{k}+d_{k}^{\lambda}\right)-\frac{1+\gamma_{h}}{2}\right. \\
& \left.\left[q_{\omega}\left(x_{k}, 0, \lambda_{k}, 0\right)-q_{\omega}\left(x_{k}, d_{k}, \lambda_{k}, d_{k}^{\lambda}\right)\right]\right\} \\
& \geq \frac{1-\gamma_{h}}{4} \kappa \theta_{k}^{2}-\kappa_{5} \theta_{k}+\left(\frac{1-\gamma_{h}}{4} \omega-\kappa_{6}\right) \\
& =\frac{1-\gamma_{h}}{4} \kappa\left[\theta_{k}-\frac{2 \kappa_{5}}{\left(1-\gamma_{h}\right) \kappa}\right]^{2}+\frac{1-\gamma_{h}}{4} \omega-\kappa_{6} \\
& -\frac{\kappa_{5}^{2}}{\left(1-\gamma_{h}\right) \kappa} .
\end{aligned}
$$

Define $\gamma_{\omega}$ : $=4 \kappa_{6} / 1-\gamma_{h}+4 \kappa_{5}^{2} /\left(1-\gamma_{h}\right)^{2} \kappa$. We conclude that (22) holds for $\omega \geq \gamma_{\omega}$.

From [7], we can easily prove that Lemma 3 is true for the next three choices of steps.

$$
\begin{gathered}
\bar{d}_{k}=d_{k}+d_{k+1}, \bar{d}_{k}^{\lambda}=d_{k}^{\lambda}+d_{k+1}^{\lambda}, \\
\bar{d}_{k}=d_{k}+d_{k+1}+d_{k+2}, \bar{d}_{k}^{\lambda}=d_{k}^{\lambda}+d_{k+1}^{\lambda}+d_{k+2}^{\lambda}, \\
\text { or } \bar{d}_{k}=d_{k}+d_{k+1}+d_{k+2}+d_{k+3}, \bar{d}_{k}^{\lambda}=d_{k}^{\lambda}+d_{k+1}^{\lambda}+d_{k+2}^{\lambda}+d_{k+3}^{\lambda} .
\end{gathered}
$$

Using arguments similar to those in the proof of Lemma 4.5, Lemma 4.6, and Theorem 4.7 in [7], we are able to show the main local convergence theorem.

Theorem 1. Suppose Assumptions L hold. For $k$ sufficiently large, the sequence $\left\{x_{k}\right\}$ converges to $x^{*}$ with a superlinear rate.

\section{Numerical Results}

We have performed numerical experiments on a personal computer with Intel CORE i5-6200U/4GB/1TB. The numerical results have been obtained by running our algorithms on the set of 43 equality constrained problems. These problems were taken from the following web page: https:// www.gamsworld.org/performance/princetonlib/htm/ group5stat.htm.

In Table 1, Name, $N$, and $M$ stand for the name of the chosen test problem, the number of variable, and the number of constraint, respectively.

We used the starting point supplied with these problems, wrote all codes in the Matlab code, and limited all attempts to solve these test problems to a maximum of 1000 iterations.

\begin{tabular}{|c|c|c|}
\hline Name & $N$ & $M$ \\
\hline eigena2 & 110 & 55 \\
\hline eigencco & 30 & 14 \\
\hline fccu & 19 & 8 \\
\hline hs007 & 2 & 1 \\
\hline hs039 & 4 & 2 \\
\hline hs077 & 6 & 2 \\
\hline hs08 & 2 & 2 \\
\hline hs046 & 5 & 2 \\
\hline hs 050 & 5 & 2 \\
\hline hs056 & 7 & 4 \\
\hline bt2 & 3 & 1 \\
\hline bt6 & 5 & 2 \\
\hline hs100lnp & 7 & 2 \\
\hline bt12 & 5 & 3 \\
\hline mwright & 5 & 3 \\
\hline eigenaco & 110 & 55 \\
\hline hatfldg & 25 & 24 \\
\hline genhs 28 & 10 & 8 \\
\hline hs027 & 3 & 1 \\
\hline hs040 & 4 & 3 \\
\hline hs078 & 5 & 3 \\
\hline hs026 & 3 & 1 \\
\hline hs048 & 5 & 2 \\
\hline hs051 & 5 & 3 \\
\hline hs061 & 3 & 2 \\
\hline bt3 & 5 & 3 \\
\hline bt7 & 5 & 3 \\
\hline bt10 & 2 & 2 \\
\hline bt5 & 3 & 2 \\
\hline eigenbco & 110 & 55 \\
\hline robot & 14 & 9 \\
\hline hs111lnp & 10 & 3 \\
\hline hs028 & 3 & 1 \\
\hline hs047 & 5 & 3 \\
\hline hs079 & 5 & 3 \\
\hline hs042 & 4 & 2 \\
\hline hs049 & 5 & 2 \\
\hline hs052 & 5 & 3 \\
\hline hs009 & 2 & 1 \\
\hline bt4 & 3 & 2 \\
\hline bt9 & 4 & 2 \\
\hline bt11 & 5 & 3 \\
\hline maratos & 2 & 1 \\
\hline
\end{tabular}
The parameters in these algorithms were chosen as follows: $\beta=0.3, \quad \gamma_{h}=0.01, \quad \gamma_{\mathscr{L}}=0.5, \quad \tau_{1}=1, \quad \nu=10^{4}, \quad s_{h}=1.5$, $s_{\mathscr{L}}=3.2, \gamma_{\alpha}=0.5$, and $\varsigma=0.5$, which seemed to work reasonably well for a broad class of problems. Update the Hessian matrix approximation $W_{k}$ by the BFGS secant update formula
TABLE 1: Numerical comparisons.

$$
W_{k+1}=B\left(u_{k}, y_{k}, W_{k}\right)=W_{k}+\frac{y_{k} y_{k}^{T}}{y_{k}^{T} u_{k}}-\frac{\left(W_{k} u_{k}\right)\left(W_{k} u_{k}\right)^{T}}{u_{k}^{T} W_{k} u_{k}}
$$

The computation terminated if the following stopping criterion was satisfied:

$$
\left\|\left(\begin{array}{c}
\nabla_{x} \mathscr{L}\left(x_{k}, \lambda_{k}\right) \\
c_{k}
\end{array}\right)\right\| \leq 10^{-5} .
$$

We summarize the numerical results in Figures 1(a)1(d) in terms of the performance profiles as outlined in [11]. Our profiles are based on the number of iterations, that of evaluating function, that of augment of the filter set, and the CPU times (seconds). The value of $\rho(\tau)$ for $\tau=1$ represents 

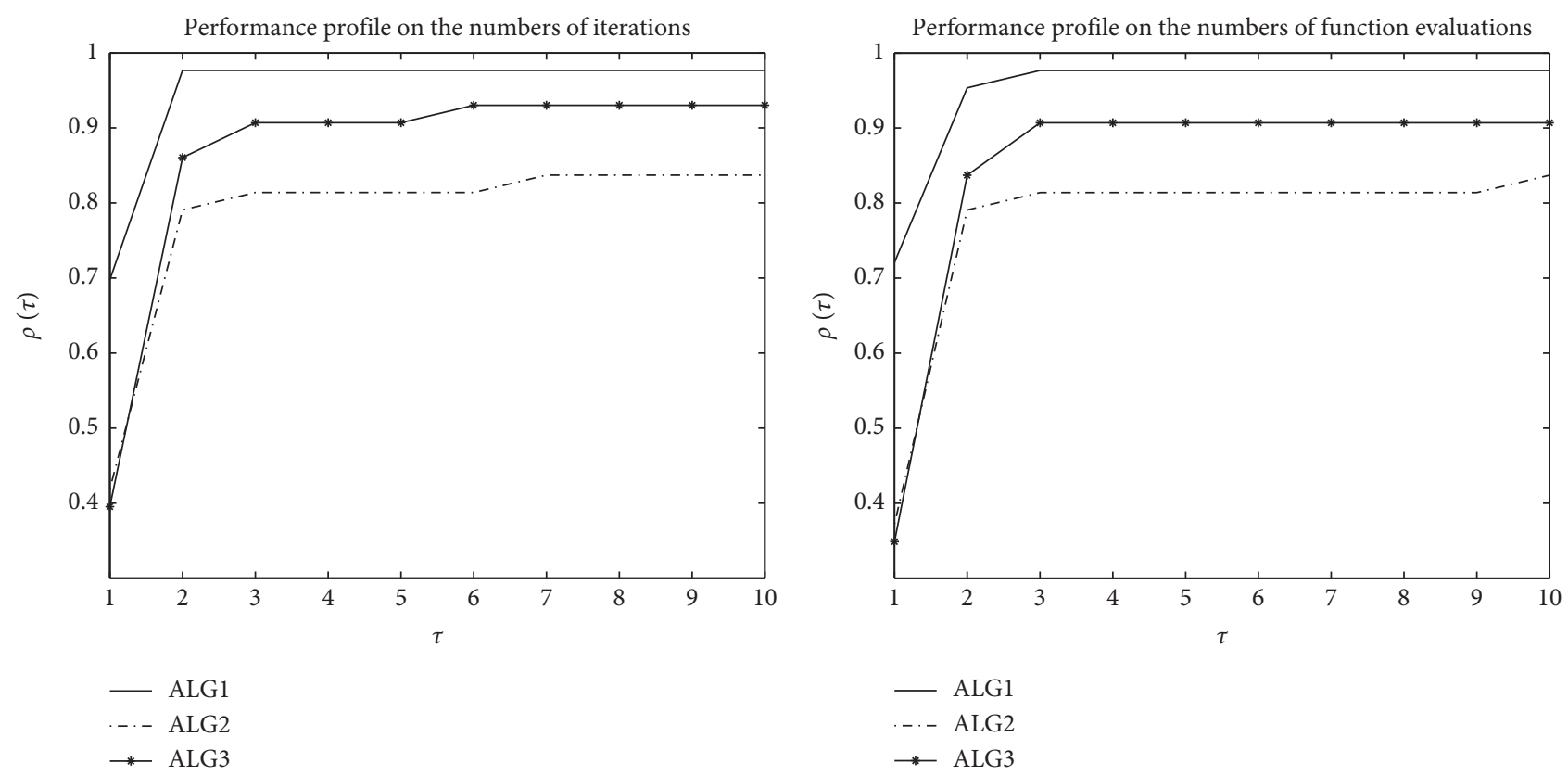

(a)
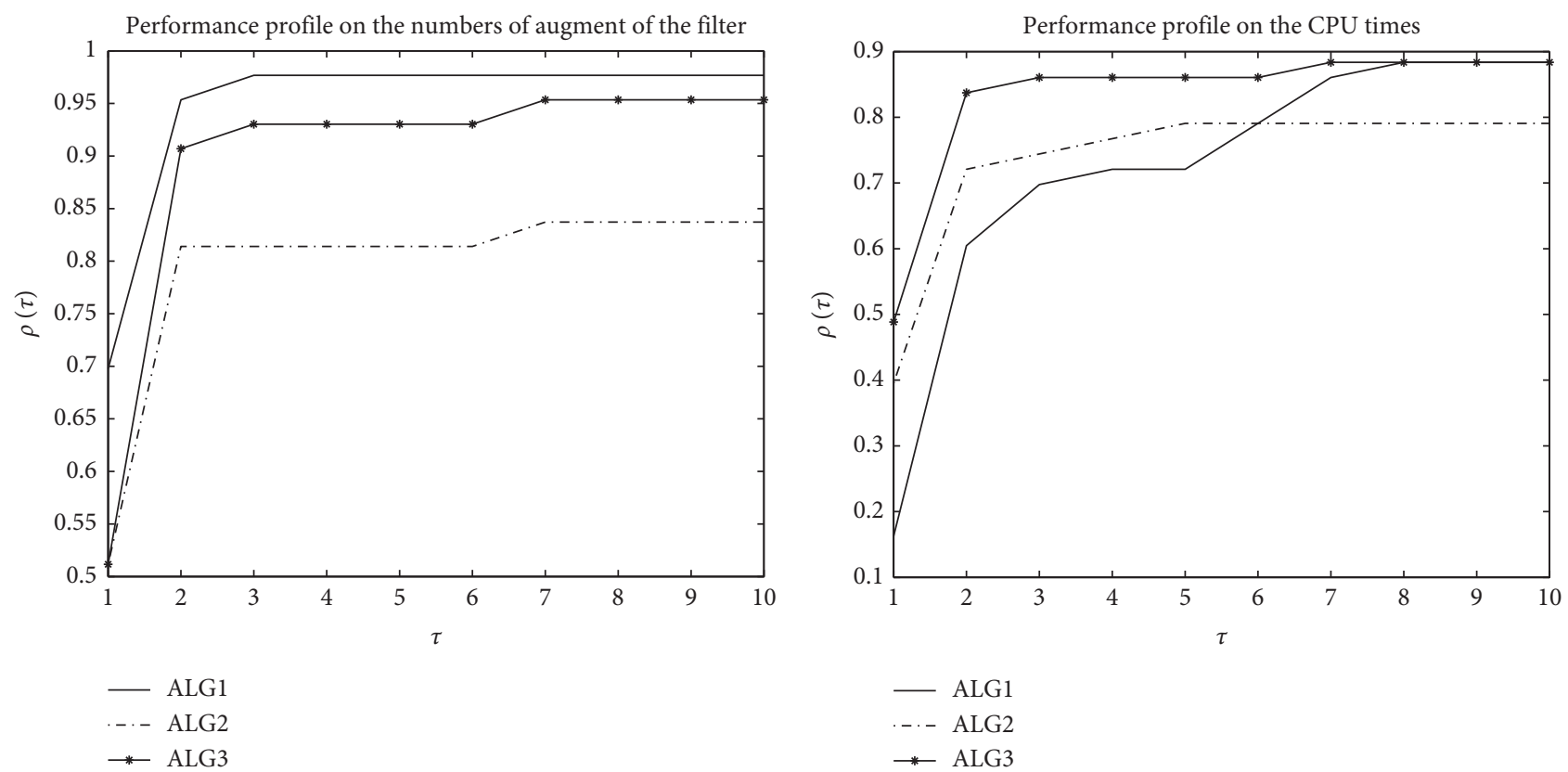

(c)

(d)

Figure 1: Performance profile on $[1,10]$.

the probability that the algorithm will win over the others. Function values $\rho(\tau)$ on the rightmost values illustrate the robustness of the approach.

Figures 1(a) and 1(b) show the performance profiles for the number of iterations and that of evaluating function required to solve the problems, by the three different algorithms. We can observe from the two figures that ALG 1, which solves $98 \%$ of the problems, is superior to the next two. Because of solving $83 \%$ of those, ALG 2 is inferior to ALG 3, which solves $92 \%$ of those. From this figure, it is clear that ALG 1 has the most wins (has the highest probability of being the optimal algorithm) and that the probability that ALG 1 is the winner on a given problem is about 0.7 .

As is known to all, the fewer number the filter set augments, the better performance the algorithm achieves. From Figure 1(c), we may analyze that ALG 1 is the most efficient on almost $70 \%$ of the problems when the number of the filter expansions is under comparison. Observing the right side of the plots in the figure, we conclude that ALG 1 solves most problems to optimality (approximately $97.5 \%$ ). 
A CPU-time performance profile is presented in Figure 1(d) for the given test problems and these 3 algorithms. An observation that emerges from the figure is that ALG 3 clearly outperforms the other algorithms. The second conclusion is that ALG 1 is by far the worst with $\tau \leq 6$. Another point of interest is that ALG 1 and ALG 3 have the best probability $\rho(\tau)$ for $\tau$ in the interval $\tau \geq 8$, with similar performance.

These performance profiles can indicate that the proposed three algorithms are effective and robust.

\section{Conclusions}

In this paper, we propose a class of inexact secant line search filter methods for solving nonlinear equality constrained optimization. The local convergence properties and numerical performance of the class of algorithms are presented. A simple modification of these methods proposed in a companion paper [7] avoiding second-order correction steps is given. Our methods do not suffer from the Maratos effect by using Lagrangian function $\mathscr{L}$ as the objective function and $\|c(x)\|^{2}$ as the constraint violation. We compare these three methods, and detailed numerical results indicate that the proposed algorithms are robust and effective, while ALG 1 is superior to the next two.

\section{Data Availability}

The data supporting the findings of this study are included within the article.

\section{Conflicts of Interest}

The authors declare that they have no conflicts of interest.

\section{Acknowledgments}

This study was supported by Scientific Research Fund of Hunan Provincial Education Department (no. 18A351).

\section{References}

[1] F. Arzani and M. R. Peyghami, "An approach based on dwindling filter method for positive definite generalized eigenvalue problem," Computational and Applied Mathematics, vol. 37, no. 2, pp. 1197-1212, 2018.

[2] R. H. Byrd, F. E. Curtis, and J. Nocedal, "An inexact SQP method for equality constrained optimization," SIAM Journal on Optimization, vol. 19, no. 1, pp. 351-369, 2008.

[3] G. Eichfelder, K. Klamroth, and J. Niebling, "Nonconvex constrained optimization by a filtering branch and bound," Journal of Global Optimization, vol. 80, no. 1, pp. 31-61, 2021.

[4] C. Gu, D. Zhu, and Y. Pei, "A new inexact SQP algorithm for nonlinear systems of mixed equalities and inequalities," Numerical Algorithms, vol. 78, no. 4, pp. 1233-1253, 2018.

[5] D. Li and D. Zhu, "An affine scaling interior trust-region method combining with line search filter technique for optimization subject to bounds on variables," Numerical Algorithms, vol. 77, no. 4, pp. 1159-1182, 2018.

[6] A. Wächter and L. T. Biegler, "Line search filter methods for nonlinear programming: motivation and Global convergence," SIAM Journal on Optimization, vol. 16, no. 1, pp. 1-31, 2005.

[7] A. Wächter and L. T. Biegler, "Line search filter methods for nonlinear programming: local convergence," SIAM Journal on Optimization, vol. 16, no. 1, pp. 32-48, 2005.

[8] R. Fontecilla, "Local convergence of secant methods for nonlinear constrained optimization," SIAM Journal on $\mathrm{Nu}$ merical Analysis, vol. 25, no. 3, pp. 692-712, 1988.

[9] R. S. Dembo, S. C. Eisenstat, and T. Steihaug, "Inexact Newton methods," SIAM Journal on Numerical Analysis, vol. 19, no. 2, pp. 400-408, 1982.

[10] Z. Wang, L. Cai, and D. Zhu, "Line search filter inexact secant methods for nonlinear equality constrained optimization," Applied Mathematics and Computation, vol. 263, no. 2, pp. 47-58, 2015.

[11] E. D. Dolan and J. J. Moré, "Benchmarking optimization software with performance profiles," Mathematical Programming, vol. 91, no. 2, pp. 201-213, 2002. 